\title{
Avaliação do efeito farmacológico de Hyptis suaveolens(L) Poit (Lamiaceae) sobre o terceiro estádio larval (L3) de Cochliomyia hominivorax e Musca domestica
}

Evaluation of the pharmacological effect of Hyptis suaveolens (L) Poit (Lamiaceae) on the third larval stage (L3) of Cochliomyia hominivorax and Musca domestica)

\section{Raylson Pereira de Oliveira ${ }^{3}$, Jeane Gomes de Matos ${ }^{1}$, Cosme Nogueira da Silva ${ }^{2}$, João Sammy Nery de Souza ${ }^{4}$, Nair Silva Cavalcanti de Lira ${ }^{6}$, Pollyana Oliveira da Silva ${ }^{5}$, Jamylla Mirck Guerra de Oliveira ${ }^{6}$, Márcia Paula Oliveira Farias ${ }^{6} *$}

Resumo: As infestações por moscas quando não controladas adequadamente podem reduzir drasticamente a produtividade dos animais e causar danos à saúde de humanos. Com o aparecimento de insetos resistentes, tem-se buscado novas bases farmacológicas a partir de plantas medicinais com alta atividade inseticida. Assim, objetivou-se com este trabalho avaliar efeito larvicida do extrato etanólico das folhas de Hyptis suaveolens (L) Poit (Lamiaceae) sobre o terceiro estádio larval (L3) de Cochliomyia hominivorax e Musca domestica. De acordo com os resultados, o extrato etanólico de $H$. suaveolens nas concentrações utilizadas de 1,0\%,10\%, 100\% e 1000\% não apresentou atividade biológica eficaz sobre as larvas de terceiro estádio de Cochliomyia hominivorax e Musca domestica.

Palavras-chave: Moscas, larvicida, extrato etanólico, fitoterápico

Abstract: Fly infestations if not properly controlled can drastically reduce the productivity of animals and harm human health. The emergence of resistant insects leads to novel pharmacological bases research on medicinal plants with high insecticidal activity. Thus, the objective of this study was to evaluate the larvicidal effect of the ethanolic extract of Hyptis suaveolens (L) Poit (Lamiaceae) leaves on the third larval stage (L3) of Cochliomyia hominivorax and Musca domestica. According to the results, the ethanolic extract of $H$. suaveolens in the concentrations of $1.0 \%, 10 \%, 100 \%$, and $1000 \%$ did not present effective biological activity on third-stage larvae of Cochliomyia hominivorax and Musca domestica.

Keywords: Fly, larvicide, ethanolic extract, herbal medicine

Autor para correspondência: E-mail: raylson.oliveira@ @otmail.com.br*

Recebido em 20.01.2020. Aceito em 30.03.2020

http://dx.doi.org/10.5935/1981-2965.20200014

${ }^{1}$ Doutorando em Biociência Animal pela pela Universidade Federal Rural de Pernambuco, Campus Recife, Departamento de Medicina Veterinária, Recife, Pernambuco, Brasil. E-mail: raylson.oliveira@hotmail.com.br*

${ }^{2}$ Médica veterinária pela Universidade Federal do Piauí, Campus professora Cinobelina Elvas, Bom Jesus, Piauí, Brasil. E-mail: jeanegomesdematos@gmail.com

${ }^{3}$ Graduando em Medicina Veterinária pela Universidade Federal da Bahia, Campus Ondina, Salvador, Bahia, Brasil. E-mail: silva5nds@ hotmail.com

${ }^{4}$ Docente da Universidade Federal do Piauí, Campus professora Cinobelina Elvas, Curso de Ciências Biológicas, Bom Jesus, Piauí, Brasil. E-mail: sammynery@yahoo.com.br

${ }^{5}$ Docente da Universidade Federal do Piauí, Campus professora Cinobelina Elvas, Curso de Zootecnia, Bom Jesus, Piauí, Brasil. E-mail: pollyanaodasilva@ hotmail.com

${ }^{6}$ Docente da Universidade Federal do Piauí, Campus professora Cinobelina Elvas, Curso de Medicina Veterinária, Bom Jesus, Piauí, Brasil. E-mail: naircl@ hotmail.com 
de Oliveira et al., Revista Brasileira de Higiene e Sanidade Animal (v.14, n. 2) p. 167 - 173 abr - jun (2020)

${ }^{7}$ Docente da Universidade Federal do Piauí, Campus professora Cinobelina Elvas, Curso de Medicina Veterinária, Bom Jesus, Piauí, Brasil. E-mail: jamyllaguerra@gmail.com

${ }^{8}$ Docente da Universidade Federal do Piauí, Campus professora Cinobelina Elvas, Curso de Medicina Veterinária, Bom Jesus, Piauí, Brasil. E-mail: marciapbo@gmail.com

\section{Introdução}

As doenças parasitárias, mesmo com avanços da medicina veterinária e tecnologias de criação de animais, continuam sendo uma das mais importantes causas de entrave ao desenvolvimento pecuário em todas as regiões brasileiras, inclusive no Nordeste. Dentre os principais problemas parasitários na pecuária inclui-se, infestações por moscas, que quando não controladas adequadamente, podem reduzir drasticamente a produtividade dos animais domésticos, enfraquecendo-os e tornandoos mais susceptíveis ao ataque de doenças (HOMER \& GOMES, 1990).

\section{Cochliomyia}

hominivorax

(Coquerel, 1858), é um tipo de mosca classificada como agente causador da míiase primária obrigatória em animais de sangue quente. Destaca-se por acarretar prejuízos à bovinocultura, sendo agente nas miíases umbilicais dos bezerros, diminuindo a produção leiteira e os índices de fertilidade, taxa de conversão alimentar e o ganho de peso, podendo, eventualmente, levar os animais infestados à morte (GUIMARÃES \& PAPAVERO, 1999).
Com relação a Musca domestica (Linnaeus, 1758), a mesma apresenta importância tanto na saúde pública, quanto na saúde animal pelo fato de ser vetor mecânico de vários agentes patogênicos, dentre os quais incluem bactérias, fungos, vírus, protozoários e helmintos (THYSSEN et al., 2004). Tais condições, aliadas a suas atividades diretas de perturbação e espoliação nos animais, acarretam enormes prejuízos à produção animal (COELHO \& GRISI, 1989).

O controle de moscas que afetam negativamente a economia de um país tem sido preocupação de muitos pesquisadores (COELHO \& GRISI, 1989). Com o aparecimento de insetos resistentes, tem-se buscado novas bases farmacológicas que apresentem como características principais, alta atividade inseticida, boa rentabilidade e baixa toxidade para mamíferos de modo a contribuir efetivamente no controle desses insetos (DO PRADO, 2003).

$\mathrm{O}$ interesse pela pesquisa das plantas medicinais vem crescendo nos últimos anos, principalmente, no que se refere à sua atividade farmacêutica e composição química. A Hyptis suaveolens (L.) Poit. (Lamiaceae) é uma erva ereta 
de Oliveira et al., Revista Brasileira de Higiene e Sanidade Animal (v.14, n. 2) p. 167 - 173 abr - jun (2020)

anual, conhecida no Brasil como bamburral (nordeste) e erva canudo (sudeste e sul), sendo muito utilizada na medicina popular para diversos fins. como carminativa e para o tratamento de distúrbios gastrintestinais (Grassi et al., 2005); antisséptica, antibacteriana (Rojas et al., 1992); antifúngica (Malele et al., 2003); larvicida contra Aedes aegypti, no quarto estádio larval (L4) (Noegroho, S. P. \& Srimulyani, 1997); anticonvulsivante (Akah \& Nwambie, 1993) e nematicida (BABU \& SUKUL, 1990).

Arivoli \& Samuel (2011) avaliando a ação larvicida e ovicida de extratos $H$. suaveolens (L.) Poit. em larvas de Aedes aegypti, Anopheles stephensie, Culex quinquefasciatus, observaram que o mesmo apresenta uma importante inibição no surgimento dos adultos.

Diante do exposto e dos resultados já comprovados em relação as suas propriedades farmacológicas, este estudo teve por objetivo avaliar o efeito larvicida do extrato etanólico das folhas de $H$. suaveolens (L) Poit (Lamiaceae) sobre o terceiro estádio larval (L3) de $C$. hominivorax e M. domestica.

\section{Material e Métodos}

Obtenção do Extrato Etanólico das Folhas de Hyptis suaveolens

As folhas de $H$. suaveolens foram coletadas nas margens da rodovia que liga o município de Bom Jesus - Piauí ao
Campus Professora Cinobelina Elvas (CPCE), e levadas posteriormente ao laboratório de química orgânica. A exsicata da planta foi identificada e armazenada no "Herbário Graziela Barroso" - TEPB/UFPI, com o registro de $\mathrm{n}^{\circ} 28.826$.

No laboratório, as folhas foram secas em estufa a $40^{\circ} \mathrm{C}$ por 8 dias, em seguida foram trituradas em moinho de facas tipo Willye, modelo SL-032. O material botânico triturado teve rendimento de 1.300 gramas, sendo submetido à extração a frio em único frasco de vidro com etanol absoluto $99,5 \%$ por $72 \mathrm{~h}$, em três repetições. A solução foi agitada diariamente, durante o período de extração. $\mathrm{O}$ extrato foi filtrado em papel de filtro e destilado à temperatura de $60^{\circ} \mathrm{C}$, sob pressão reduzida de 500 a $750 \mathrm{mmHg}$, em evaporador rotativo, modelo 802, fornecendo o extrato etanólico das folhas de $H$. suaveolens. O material concentrado foi liofilizado durante $72 \mathrm{~h} \quad \mathrm{e}$ acondicionado para o uso posterior.

Atividade larvicida contra Cochliomyia hominivorax e Musca domestica

Para permitir a disponibilidade de insetos durante o período do experimento, colônias de $M$. domestica e $C$. hominivorax foram mantidas no Laboratório de Parasitologia Veterinária da UFPI-CPCE. A colônia de $C$. hominivorax foi mantida de acordo com a metodologia descrita por 
de Oliveira et al., Revista Brasileira de Higiene e Sanidade Animal (v.14, n. 2) p. 167 - 173 abr - jun (2020)

Mastrangelo (2011) e a de $M$. domestica segundo FARIAS et al. (2009).

$\mathrm{O}$ teste com $C$. hominivorax foi realizado utilizando-se a técnica descrita por Giglioti et al. (2008) com algumas adaptações, em que no presente teste foi realizado em triplicata com 4 diluições do extrato etanólico de $H$. suaveolens, nas concentrações de 1,0\%, 10\%, $100 \%$ e $1000 \%$, como controle positivo foi usado a Permetrina e como controle negativo água destilada. Para o teste com $M$. domestica foi usado a metodologia proposta por Farias et al. (2009) com as mesmas adaptações usada no teste com $C$. hominivorax.

A análise estática do efeito larvicida do extrato foi realizada utilizando o percentual médio de mortalidades em cada tratamento, de acordo com a espécies estudadas. Essas médias passaram pelo teste de Tukey $(\mathrm{P}<0,05)$, para comparação de média. Nas análises utilizou-se o comando PROC GLM do software SAS University Edition.

\section{Resultados e discussão}

Neste estudo, observou-se que o extrato etanólico das folhas de $H$. suaveolens não apresentou potencial larvicida satisfatório nas concentrações testadas, sendo observado efeito inferior a $50 \%$ em todas as concentrações testadas, tanto para larvas de terceiro estádio de $C$. hominivorax (Tabela 1), quanto para larvas de M. domestica (Tabela 2).

Esse resultado foi similar ao observado em outro estudo, em que o extrato de $H$. suaveolens não apresentou atividade repelente contra Lutzomyia migonei (NIEVES et al., 2010).

Tabela 1 - Mortalidade média de larvas de terceiro estádio de Cochliomyia hominivorax de acordo com as concentrações do extrato etanólico das folhas de Hyptis suaveolens

\begin{tabular}{lllllll}
\hline $\begin{array}{l}\text { Concentrações do extrato etanólico } \\
\text { de Hyptis suaveolens }(\mu \mathrm{g} / \mathrm{ml})\end{array}$ & $1,0 \%$ & $10 \%$ & $100 \%$ & $1000 \%$ & $\mathrm{CN}$ & $\mathrm{CP}$ \\
$\begin{array}{l}\text { Taxa (\%) de Larvas mortas } \\
13.33^{\mathrm{c}}\end{array}$ & $13.33^{\mathrm{c}}$ & $20.00^{\mathrm{c}}$ & $43.33^{\mathrm{b}}$ & $3.33^{\mathrm{c}}$ & $100.00^{\mathrm{a}}$ \\
\hline $\begin{array}{l}\text { *As médias seguidas pela mesma letra não diferem estatisticamente entre si (Teste de Tukey } \\
\text { ao nível de 5\% de probabilidade). CN: controle negativo; CP: controle positivo. }\end{array}$ &
\end{tabular}


de Oliveira et al., Revista Brasileira de Higiene e Sanidade Animal (v.14, n. 2) p. 167 - 173 abr - jun (2020)

Tabela 2 - Mortalidade média de larvas de terceiro estádio de Musca domestica de acordo com as concentrações do extrato etanólico das folhas de Hyptis suaveolens

\begin{tabular}{lllllll}
\hline $\begin{array}{l}\text { Concentrações do extrato etanólico } \\
\text { de Hyptis suaveolens }(\mu \mathrm{g} / \mathrm{ml})\end{array}$ & $1,0 \%$ & $10 \%$ & $100 \%$ & $1000 \%$ & $\mathrm{CN}$ & $\mathrm{CP}$ \\
& & & & & & \\
Taxa (\%) de Larvas mortas & $23.33^{\mathrm{b}}$ & $26.66^{\mathrm{b}}$ & $26.66^{\mathrm{b}}$ & $33.33^{\mathrm{b}}$ & $20.00^{\mathrm{b}}$ & $53.33^{\mathrm{a}}$
\end{tabular}

*As médias seguidas pela mesma letra não diferem estatisticamente entre si, (Teste de Tukey ao nível de 5\% de probabilidade). $\mathrm{CN}$ : controle negativo; $\mathrm{CP}$ : controle positivo.

O resultado não responsivo do extrato etanólico da $H$. suaveolens pode estar associado com o solvente utilizado no presente estudo, pois como mostrado no trabalho do Yadav et al. (2015), a concentração mínima de eficácia pode variar de acordo com o solvente utilizado para a produção do extrato e também com o período do ano de coleta da planta.

Outra possibilidade para o resultado apresentado seria a região da coleta, pois como mostrado em outros trabalhos, os componentes da $H$. suaveolens podem variar de acordo com a latitude de um estado, sendo mais predominante certos tipos de componentes no extrato que outros, como sesquiterpenes (MARTINS et al., 2006). Também pode ser observada essa diferença quando comparado os componentes da $H$. suaveolens entre os países, sendo possível realizar o mesmo experimento com a planta originada de outro país (MCNEIL et al.,, 2011).

Essa variação dos componentes da H. suaveolens é de grande importância, já que a presença em maior ou menor concentração de um componente específico, como polissacarídeos neutros, pode alterar a atuação dessa planta como um probiótico, assim como a menor concentração de $\alpha$-humuleno pode alterar a atuação repelente a carrapaticidas (GRASSI et al., 2005). Dessa forma, é possível que a atividade larvicida não apresentada no estudo esteja associada com a composição da $H$. suaveolens da região.

Os resultados apresentados não se enquadram nos padrões estabelecidos pelo Ministério da Agricultura, conforme legislação pertinente à comercialização de produtos com atividade larvicida no País (BRASIL., 1990), que é de 95\%, uma vez que os resultados obtidos em cada um dos testes foram inferiores a 50\%. Foi possível observar também que larvas de $C$. hominivorax são sensíveis à Permetrina, apresentando uma mortalidade de 100\%, princípio ativo que foi utilizado como controle positivo. Já larvas de terceiro estádio de $M$. domestica apresentaram 
de Oliveira et al., Revista Brasileira de Higiene e Sanidade Animal (v.14, n. 2) p. 167 - 173 abr - jun (2020)

resistência ao fármaco, com taxa de mortalidade $53,33 \%$, sendo observada uma eficácia inferior ao preconizado pelo Ministério da Agricultura (BRASIL., 1990).

Essa resistência da $M$. domestica pode ser causada por vários fatores, um deles devido a relação de proximidade que as mesmas mantem com seres humanos, estando em contato direto com dejetos, ou até mesmo com inseticidas químicos, que podem levar a seleção de genes necessários para o desenvolvimento de resistência aos produtos atuais mais poderosos (DO PRADO, 2003).

Embora o presente estudo não tenha demonstrado a atividade larvicida do extrato etanólico de $H$. suaveolens (bamburral) sobre Larvas de terceiro estádio de $C$. hominivorax e $M$. domestica, outros estudos observaram que o óleo essencial desta planta possui ação antiséptica, antibacteriano, anticarcinogênico, antifúngica e larvicida contra Aedes aegypti (MARTINS et al., 2006).

\section{Conclusão}

O extrato etanólico de $H$. suaveolens nas concentrações utilizadas de $1,0 \%, 10 \%, 100 \%$ e $1000 \%$ não apresenta atividade biológica eficaz sobre as larvas de terceiro estádio de Cochliomyia hominivorax e Musca domestica.

\section{Referências bibliográfica}

1. AKAH, P. A.; NWAMBIE, A. I. Nigerian plants with anti-convulsant property. Fitoterapia, v. 64, n. 1, p. 42-44, 1993.

2. ARIVOLI, S.; SAMUEL, T. Mosquitocidal activity of Hyptis suaveolens (L) Poit (Lamiaceae) extracts against Aedes aegypti, Anopheles stephensi and Culex quinquefasciatus (Diptera: Culicidae). International Journal of Recent Scientific Research, v. 2, n. 5, p. 143-149, 2011.

3. BABU, S.P.S.; SUKUL, N.C. Essential oils as nematicidal principles. Environment and Ecology, v. 8, n. 4, p. 1118-1120, 1990.

4. BRASIL., 1990. Ministério da agricultura. Portaria n. 90 de 04 de dezembro de 1989. Normas para produção, controle e utilização de produtos antiparasitários. Diário Oficial, p. sec. 1, 2. Edição de 22/01/1990., 1990.

5. COELHO, V.M.A.; GRISI, L. Técnica para avaliação da atividade de inseticida contra Musca domestica(L.) e Stomoxys calcitrans (L.) em bovinos. Arquivo Universidade Federal Rural do Rio de Janeiro, v. v. 12, n. n.1-2, p. 33-40, 1989.

6. DO PRADO, A.P. Controle das principais espécies de moscas em áreas urbanas. Biológico, v. 65, n. 1-2, p. 95-97, 2003.

7. FARIAS, M.P.O.; DE BARROS, F.N.; ALVES, L.C.; FAUSTINO, M.A. DA G. . EFICÁCIA DO ÓLEO DA SEMENTE DE ANDIROBA ( Carapa guianensis ) SOBRE LARVAS DE Musca domestica ( DIPTERA : MUSCIDAE ) POR. p. 3-5, [s.d.]. 
8. GIGLIOTI, R.; CARVALHO, C.O.; FERREZINI, J.; OLIVEIRA, M.C. DE S.; CHAGAS, A. C. S. Efeito do óleo essencial de. p. 4, 2011.

9. GRASSI, P. et al. Chemical polymorphism of essential oils of Hyptis suaveolens from El Salvador. Flavour and Fragrance Journal, v. 20, n. 2, p. 131$135,2005$.

10. GUIMARÃES, J.H.; PAPAVERO, N. Myiasis in man and animals in the neotropical region. Pleiade I FAPESP, v. cap, v. 1, p. 15-18, 1999.

11. HONER, M.R. \& GOMES, A. O manejo integrado de mosca dos chifres, berne e carrapato em gado de corte. Campo Grande, EMBRAPA-CNPGC, 1990. 60p. (EMBRAPA-CNPGC. Circular Técnica, 22).

12. MALELE, R.S. et al. Essential Oil of Hyptis suaveolens (L.) Poit. from Tanzania: Composition and Antifungal Activity. Journal of Essential Oil Research, v. 15, n. 6, p. 438-440, 1 nov. 2003.

13. MARTINS, F.T. et al. Variação química do óleo essencial de Hyptis suaveolens (L.) poit., sob condições de cultivo. Quimica Nova, v. 29, n. 6, p. 1203-1209, 2006.

14. MASTRANGELO, T. DE A. UNIVERSIDADE DE SÃO PAULO CENTRO DE ENERGIA NUCLEAR NA AGRICULTURA THIAGO DE ARAÚJO MASTRANGELO Metodologia de produção de moscas estéreis de. 2011. 15. MCNEIL, M.; FACEY, P.; PORTER, R. Essential oils from the Hyptis genus - A review (1909-2009). Natural Product Communications, v. 6 , n. 11 , p. 17751796, 2011.
16. NIEVES, E. et al. Actividad repelente de aceites esenciales contra las picaduras de Lutzomyia migonei (Diptera: Psychodidae). Revista de Biologia Tropical, v. 58, n. 4, p. 1549-1560, 2010.

17. NOEGROHO, S.P.; SRIMULYANI, M.B. Larvicidal activity of Hyptis suaveolens essential oil toward 4th instar Aedes aegypti larvae and gas chromatographic-mass spectroscopic analysis of the oil. Majalah Farmasi Indonesia, v. 8, p. 160-170, 1997.

18. ROJAS, A. et al. Screening for antimicrobial activity of crude drug extracts and pure natural products from Mexican medicinal plants. Journal of Ethnopharmacology, v. 35, n. 3, p. 275283, 1992.

19. THYSSEN, P.J. et al. The role of insects (Blattodea, Diptera, and Hymenoptera) as possible mechanical vectors of helminths in the domiciliary and peridomiciliary environment. Cadernos de saúde pública / Ministério da Saúde, Fundação Oswaldo Cruz, Escola Nacional de Saúde Pública, v. 20, n. 4, p. 1096-1102, 2004.

20. YADAV, R. et al. Screening of some weeds for larvicidal activity against Aedes albopictus, a vector of dengue and chikungunya. n. March, p. 88-94, 2015. 